\title{
IMAGE DEBLURRING IN BAYESIAN FRAMEWORK USING TEMPLATE BASED BLUR ESTIMATION
}

\author{
Sitara K and Remya S \\ Department of Computer Science, University of Kerala, Kariavattom, \\ Thiruvananthapuram, Kerala, India \\ sitarak1987@gmail.com \\ remyayes@gmail.com
}

\begin{abstract}
Image deblurring aims to reconstruct a high quality image from a degraded image. For deblurring, the blurring function which causes the degradation is estimated first. A template based blur estimation method is presented here which can identify Gaussian and Uniform blur. Due to the ill-posed nature of image restoration (IR) process, prior knowledge of natural images is used to regularize the IR problem. The Bayesian approach provides the means to incorporate prior knowledge in data analysis. A comparative analysis using various priors was studied qualitatively. PSNR, SNR, SSIM and IQI are the performance measures used.
\end{abstract}

\section{KEYWORDS}

Image Restoration, Point Spread Function, Image Deblurring, Prior

\section{INTRODUCTION}

Images captured in uncontrolled environments invariably represent a degraded version of an original image due to imperfections in the imaging and capturing process. This degradation may be classified into two major categories: blur and noise. Images may be blurred due to atmospheric turbulence, defocusing of the lens, aberration in the optical systems, relative motion between the imaging system and the original scene. Automatic image deblurring is an objective of great practical interest for the enhancement of images in photo and video cameras, in astronomy, in tomography, in other biomedical imaging techniques, motion tracking applications, etc.

Image deblurring is an inverse problem whose aim is to recover an image from a version of that image which has suffered a linear degradation, with or without noise. In this paper, it tries to recover the original, sharp image by using a mathematical model of the blurring process. Some information on the lost details is indeed present in the blurred image-but this information is "hidden" and can only be recovered if the details of the blurring process are known. Due to various unavoidable errors in the recorded image, the original image cannot be recovered exactly. This blurring degradation can be shift-variant or shift-invariant. This work focuses on shiftinvariant blurs, and so here, "blur" will refer to a linear, shift-invariant degradation, i.e., a convolution, with or without noise, unless stated otherwise. Image deblurring methods can be divided into two classes: nonblind and blind. In nonblind deblurring methods, the blurring operator is assumed to be known. Blind deconvolution refers to a class of problems when the original image is estimated from the degraded observations where the exact information about the

DOI : 10.5121/ijma.2012.4112 
degradation and noise is not available. The blind deconvolution problem is very challenging since it is hard to infer the original image and the unknown degradation only from the observed image. The method that is described here belongs to the latter class. In most situations of practical interest the blurring filter's impulse response, also called point spread function (PSF), is not known with good accuracy. Since nonblind deblurring methods are very sensitive to mismatches between the PSF used by the method and the true blurring PSF, a poor knowledge of the blurring PSF normally leads to poor deblurring results. In blind image deblurring (BID), not only the degradation operator is ill-conditioned, but the problem also is severely ill-posed as the uniqueness and stability of the solution is not guaranteed: there are infinite number of solutions (original image + blurring filter) that are compatible with the degraded image. An overview of BID methods can be obtained from [1]. Most of the methods to solve it are iterative, and, therefore they are computationally costly. Due to the presence of noise they suffer from the stability and convergence problems.

In some cases, one has access to more than one degraded image from the same original scene, a fact which can be used to reduce the ill-posedness of the problem [13], [1]. In [13] they presented a novel filtering method for reconstructing an all-in-focus image or an arbitrarily focused image from two images that are focused differently. Karen et. al[14] proposed a novel nonparametric regression method for deblurring noisy images based on the local polynomial approximation (LPA) of the image and the intersecting confidence intervals (ICI) that is applied to define the adaptive varying scales (window sizes) of the LPA estimators. Lokhande, Arya and Gupta [16] proposed a technique for restoring the motion blurred images.

In this work, first the blur model and its parameters are identified from the blurred image to restore the image. Here only Gaussian blur and Uniform blur are considered. A novel method has been proposed for this task. This is based on the specific distortions caused by the distorting operator in the Fourier spectrum amplitude of an image. A template based on several blurred images is constructed for both Gaussian and uniform blur. The Fourier spectrum amplitude of the noisy blurred image is compared with the templates to identify the blur model. The parameters of blur are also determined from the Fourier spectrum amplitude thresholded image. With this estimated blur model and parameter values, the blurring filter PSF is constructed, which is used for obtaining the image estimate (deblurred image) from the blurred image. Due to the ill-posed nature of IR, prior knowledge of natural images can be used to regularize the IR problem. The Bayesian approach [2] provides the means to incorporate prior knowledge in data analysis. The priors used here are Tikhonov (L2), Sobolev, Total Variational (TV) [3], Sparsity [4] and the Sparse and Redundant prior [5].

The system is implemented and experiments were conducted with blur parameter set as 7,9 and 11 for Uniform blur and parameters 2.5, 2.9 and 3.3 for Gaussian blur. The performance measures used are Peak signal-to-noise ratio (PSNR), Signal-to-noise ratio (SNR), Structural similarity index measure (SSIM) and Image quality index (IQI). From the results obtained it can be inferred that the sparse and redundant prior (ASDS method) gives better results.

\section{Image Deblurring Problem}

The image degradation is modeled by

$$
y=h * x+w
$$

in which $y$ and $x$ are images which represent, respectively, the degraded image, the original image and $w$ is the additive noise (white gaussian noise is taken); $h$ is the PSF of the blurring operator, and $*$ denotes the mathematical operation of convolution. An alternative way of describing (1) is 
through its spectral equivalence. By applying discrete Fourier transforms to (1), the following representation is obtained:

$$
Y=H X+W
$$

where capitals represent Fourier transforms of $y, x, h$ and $w$. In practice the spectral representation is more often used since it leads to efficient implementations of restoration filters in the (discrete) Fourier domain. If deblurring is obtained by dividing the Fourier transform by the blurring filter PSF, this will lead to the explosion of noise.

Prior knowledge of natural images is used to regularize the IR problem. The Bayesian approach [2] provides the means to incorporate prior knowledge in data analysis. Bayesian analysis revolves around the posterior probability, which summarizes the degree of one's certainty concerning a given situation. Bayes's law states that the posterior probability is proportional to the product of the likelihood and the prior probability. The likelihood encompasses the information contained in the new data. The prior expresses the degree of certainty concerning the situation before the data are taken. Inorder to improve the knowledge concerning a parameter $x$ the present state of certainty is characterized by the probability density function $p(x)$. Perform an experiment and take some data $d$. Bayes's law becomes:

$$
p(x \mid d)=\frac{p(d \mid x) p(x)}{p(d)}
$$

$p(x \mid d)$ is the posterior probability density function, or simply the posterior, because it effectively follows the experiment. It is the conditional probability of $x$ given the new data $d$. The probability $p(x)$ is called the prior because it represents the state of knowledge before the experiment. The quantity $p(d \mid x)$ is the likelihood, which expresses the probability of the data $d$ given any particular $x$. The likelihood is usually derived from a model for predicting the data, given $x$, as well as a probabilistic model for the noise. The term in the denominator $p(d)$ may be considered necessary only for normalization purposes. As the normalization can be evaluated from the other terms, Bayes's law is often written as a proportionality, leaving out the denominator.

Although the posterior probability completely describes the state of certainty about any possible image, it is often necessary to select a single image as the 'result' or reconstruction. A typical choice is that image that maximizes the posterior probability, which is called the MAP (maximum a posteriori) estimate. Given the data $\mathbf{y}$, the posterior probability of any image $\mathbf{x}$ is given by Bayes's law (3) in terms of the proportionality

$$
p(x \mid y) \alpha p(y \mid x) p(x)
$$

where $p(\mathbf{y} \mid \mathbf{x})$, the probability of the observed data given $\mathbf{x}$, is the likelihood and $p(\mathbf{x})$ is the prior probability of $\mathbf{x}$. The negative logarithm of the posterior probability density function is given by

$$
\log [p(x \mid y)]=\phi(x)=\Lambda(x)+\Pi(x)
$$

where the first comes from the likelihood and the second term from the prior probability.

The likelihood is specified by the assumed probability density function of the fluctuations in the measurements about their predicted values (in the absence of noise). For the additive, uncorrelated Gaussian noise assumed, the negative log (likelihood) is just half of chi-squared which is quadratic in the residuals.

$$
-\log [p(\mathbf{y} \mid \mathbf{x})]=\Lambda(\mathbf{x})=\frac{1}{2} \chi^{2}=\frac{1}{2 \sigma_{\mathbf{w}}^{2}}\|\mathbf{y}-\mathbf{h x}\|^{2}
$$


The International Journal of Multimedia \& Its Applications (IJMA) Vol.4, No.1, February 2012

The choice for the likelihood function should be based on the actual statistical characteristics of the measurement noise. The restored image can be obtained from Eq. (7).

$$
\hat{\mathbf{x}}=\underset{x}{\arg \min } \frac{1}{2}\|\mathbf{y}-\mathbf{h x}\|_{2}^{2}+\lambda \Pi(\mathbf{x})
$$

where $\hat{x}$ is the restored image or image estimate and $\lambda$ is the regularization parameter.

\subsection{Blur Model}

In this paper, only Gaussian and Uniform blurs are considered.

\subsubsection{Uniform Blur}

The uniform rectangular blur is described by the following function:

$$
h\left(n_{1}, n_{2}\right)=\left\{\begin{array}{cc}
\frac{1}{l^{2}}, & \left|n_{1}\right|<\frac{l}{2},\left|n_{2}\right|<\frac{l}{2}, \\
0, & \text { elsewhere }
\end{array}\right.
$$

where parameter $l$ defines the size of smoothing area. The frequency characteristics of Eq. (8) is shown in Figure 1.
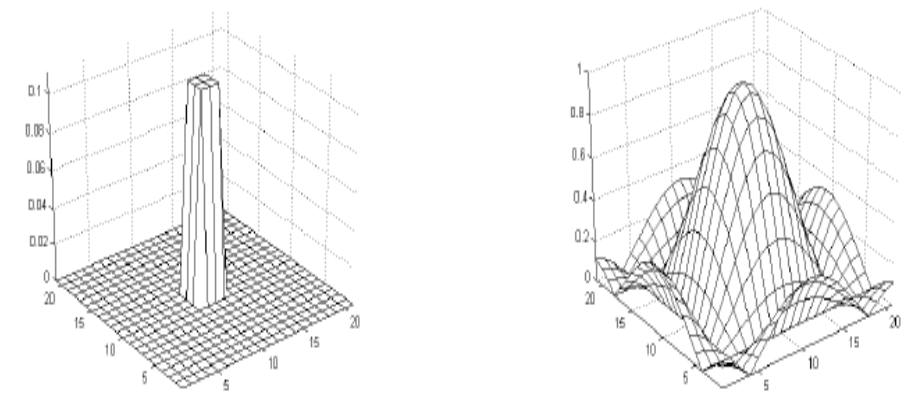

Figure 1. Uniform blur of size $3 \times 3$ and its frequency characteristics

\subsubsection{Atmospheric Turbulence Blur}
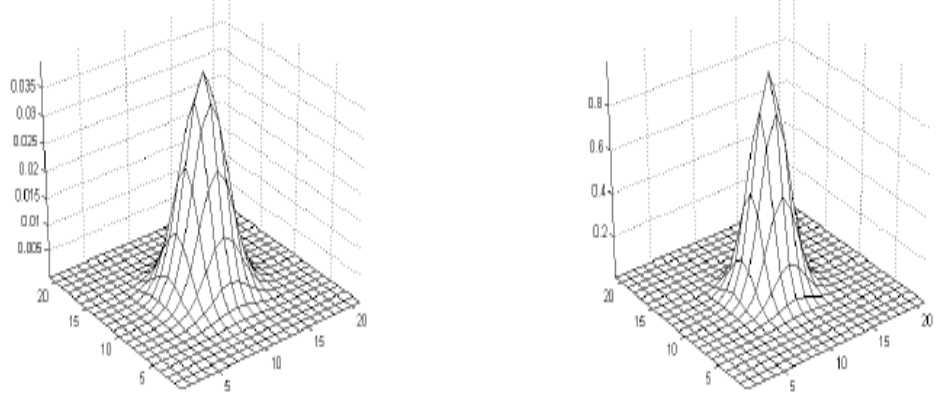

Figure 2. Gaussian PSF with $\tau=2$ and its frequency characteristics

Atmospheric turbulence is a severe limitation in remote sensing. Although the blur introduced by atmospheric turbulence depends on a variety of factors (such as temperature, wind speed, exposure time), for long-term exposures the point-spread function can be described reasonably well by a Gaussian function: 
The International Journal of Multimedia \& Its Applications (IJMA) Vol.4, No.1, February 2012

$$
h\left(n_{1}, n_{2}\right)=\frac{1}{2 \pi \tau^{2}} \exp \left(-\frac{n_{1}^{2}+n_{2}^{2}}{\tau^{2}}\right)
$$

where $\tau^{2}$ is a parameter of the PSF (the variance of the Gaussian function) (Figure 2). Its Fourier transform is also a Gaussian function and its absolute values are shown in Figure 2.

\section{ESTIMATION OF BLURRING Filter PSF}

A prior knowledge about the distorting operator and its parameters is of crucial importance in blurred image restoration. In this paper, a template based method is proposed for the identification of the type of blur and its parameters. Only Gaussian and uniform blur can be identified with this method. The Fourier spectrum of an image is influenced by the blur. This is shown in Figure 3. So here a method to identify the blur from this Fourier amplitude spectrum is proposed. After identifying the type of blur, its parameters are also extracted from the Fourier spectrum thresholded image.

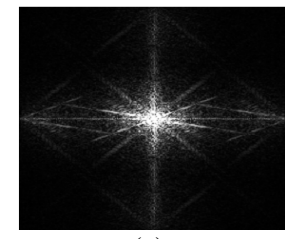

(a)

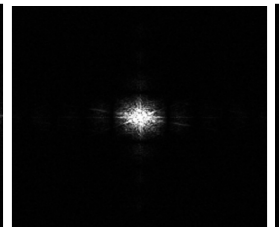

(b)

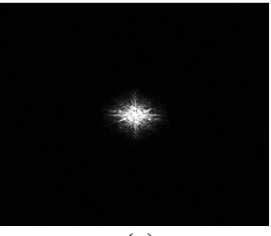

(c)

Figure 3. Influence of blur on Fourier spectrum amplitude: (a) spectrum amplitude of image that is not corrupted (b) spectrum amplitude of the same image corrupted by the uniform blur (c) spectrum amplitude of the same image corrupted by the Gaussian blur.

\subsection{Blur Template}

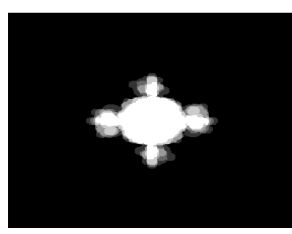

(a)

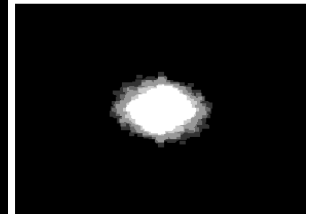

(b)

Figure 4. Blur templates: (a) Uniform blur template (b) Gaussian blur template

The blur template is constructed from the thresholded Fourier spectrum image of several blurred images. First, the Fourier spectrum amplitude of blurred images are thresholded to reduce the noise effect. After thresholding, a morphological operation [18] is performed on the resultant image. Morphological operations apply a structuring element to an input image, creating an output image of the same size. In morphological operations, the value of each pixel in the output image is based on a comparison of the corresponding pixel in the input image with its neighbors. By choosing the size and shape of the neighborhood, a morphological operation that is sensitive to specific shapes in the input image can be constructed. Here dilation is performed with the disk structuring element. A structuring element is a matrix consisting of only 0's and 1's that can have any arbitrary shape and size. The pixels with values of 1 define the neighborhood. Dilation adds pixels to the boundaries of objects in an image. The number of pixels added or removed from the objects in an image depends on the size and shape of the structuring element used to process the image. The value of the output pixel is the maximum value of all the pixels in the input pixel's neighborhood. In a binary image, if any of the pixels is set to the value 1, the output pixel is set to 1. The dilation is performed inorder to set the boundary pixels to connect all the white regions. 
The International Journal of Multimedia \& Its Applications (IJMA) Vol.4, No.1, February 2012

Several blurred images are created for both Gaussian and Uniform blur. From each of these blurred images, the dilated images are constructed. The mean image of the dilated images of Gaussian blurred images is created and this is taken as the template for identifying Gaussian blur. Similarly the template for uniform blur identification is also constructed. Thus the templates for identifying both the blurs are available (Figure 4), so by using these templates it can decide to which blur type, a new blurred image belongs.

\subsection{Blur Identification}

For deblurring, first the Fourier spectrum amplitude of the blurred image is taken. This image is thresholded and dilated. The resultant image is matched with both of the blur templates shown in Figure 4. For this, the difference image is computed. i.e., the resultant image is subtracted from each of the blur templates. Now there are two difference images corresponding to the Gaussian and Uniform blur templates. The number of non-zero elements in these difference images is computed. The type of blur template which generates the difference image whose number of nonzero elements is minimum, is identified as the blur type. If the blurred image belongs to some other blur model then the number of non-zero elements in the difference image will be very high. But still there exists a minimum value with the two blur templates. This leads to incorrect blur estimate. So to avoid this, a constraint is made that this minimum value should be less than a constant.

\subsection{Blur Parameter Estimation}

For Gaussian blur parameter estimation, the radius of the center white portion of the thresholded and dilated image of the Fourier spectrum amplitude of the blurred image is used. In the case of Gaussian blur, variance is the blur parameter. Variance has influence over the radius of the center white portion of the dilated image. i.e., the radius corresponding to a range of variance values will be different. Once the radius is computed and if it is found less than a specific value, then the variance value corresponding to that will be computed as follows:

$$
\begin{aligned}
& \text { val }=1-((\text { radius }- \text { lower_radius }) /(\text { upper_radius }- \text { lower_radius })) \\
& \text { variance }=(0.5 * \text { val })+\text { lower_variance }
\end{aligned}
$$

The radius and variance range is given in Table 1, this is taken based on several experiments. For example, if radius $>50$, the variance is computed as:

$$
\begin{aligned}
& \mathrm{val}=1-((\text { radius }-50) /(61-50)) \\
& \text { variance }=(0.5 * \text { val })+1
\end{aligned}
$$

For uniform blur, the breadth and height of the center white portion is calculated and minimum of it is taken as length. If length is in the range or less than a specific value, then the kernel size corresponding to that is selected, shown in Table 2. With this estimated blur type and blur parameter, the blurring filter PSF can be constructed as specified in section 2.1. This estimated PSF is used for deblurring the image. Image deblurring in Bayesian framework using different priors are explained in the following section.

\section{PRIORS}

The choice of prior is very important. Five priors (Tikhonov (L2), Sobolev, Total variational (TV), Sparse, Sparse and redundant prior) are taken here. 
The International Journal of Multimedia \& Its Applications (IJMA) Vol.4, No.1, February 2012

\subsection{Tikhonov and Sobolev Priors}

The Tikhonov prior is also known as the L2-norm. This prior is based on the fact that the energy present in images is bounded, but this is not the case with noise. The image estimate (deblurred image) can be obtained from the Eq. (11).

$$
\hat{x}=\underset{x}{\arg \min }\|y-h * x\|_{2}^{2}+\lambda\|x\|_{2}^{2}
$$

where $\|x\|_{2}^{2}=\left(\sum_{i}\left|x_{i}\right|^{2}\right)^{1 / 2}$. Since the filtering is diagonalized over Fourier, the solution is simply computed over the Fourier domain as:

$$
\hat{X}(\omega)=\frac{Y(\omega) \hat{H}(\omega)}{\|\hat{H}(\omega)\|^{2}+\lambda}
$$

where $\hat{X}(\omega), Y(\omega)$ and $H(\omega)$ denotes the Fourier transform of restored image, blurred image and blurring filter PSF. $\lambda$ is the regularization parameter.

Table 1. Gaussian parameter selection

\begin{tabular}{|l|l|}
\hline Radius & $\begin{array}{l}\text { Variance } \\
\text { range }\end{array}$ \\
\hline$>=61$ & 1 \\
\hline$>=50$ & $<=1.5$ \\
\hline$>=41$ & $<=2$ \\
\hline$>=36$ & $<=2.5$ \\
\hline$>=32$ & $<=3$ \\
\hline$>=29$ & $<=3.5$ \\
\hline$>=25$ & $<=4$ \\
\hline$>=23$ & $<=4.5$ \\
\hline$>=21$ & $<=5$ \\
\hline otherwise & $>=5.5$ \\
\hline
\end{tabular}

Table 2. Uniform parameter selection

\begin{tabular}{|l|l|}
\hline Length & $\begin{array}{l}\text { Kernel size } \\
\text { taken }\end{array}$ \\
\hline$>60$ & 7 \\
\hline Between 49 and 55 & 9 \\
\hline Otherwise & 11 \\
\hline
\end{tabular}

L2 regularization does not perform any denoising. So to remove noise, high frequencies in the blurred image can be penalized using the Sobolev prior. The prior is as follows:

$$
\Pi(x)=\sum_{i}\|\nabla x(i)\|^{2}=\sum_{\omega} S(\omega)\|X(\omega)\|^{2}
$$

where $S(\omega)=\|\omega\|^{2}$. Since this prior can be written over the Fourier domain, the solution to the deblurring with Sobolev prior can be simply computed with the Fourier coefficients:

$$
\hat{X}(\omega)=\frac{Y(\omega) \hat{H}(\omega)}{\|\hat{H}(\omega)\|^{2}+\lambda S(\omega)}
$$


The International Journal of Multimedia \& Its Applications (IJMA) Vol.4, No.1, February 2012

The lena image is blurred using Gaussian blur of variance 2.5. The blurred lena image is deblurred using the L2 and Sobolev priors and the resultant image is shown in Figure 5. The blur estimated is Gaussian with variance 2.3, the blurring filter constructed using this is used to deblur the image.

\subsection{Total Variation (TV) Prior}

Sobolev regularization perform a denoising but also tends to blur the edges. The TV prior [3] is able to better reconstruct sharp edges. It reads:

$$
\Pi(x)=\sum_{i}\|\nabla x(i)\|
$$

With respect to the Sobolev energy, it simply measure the L1-norm instead of the L2-norm, thus dropping the square in the functional. L1-norm is the summation of absolute values of all the image intensity elements. Unfortunately, the TV functional $\Pi(x)$ is not a smooth function of the image $x$. It thus requires the use of advanced convex optimization method to be minimized for regularization. An alternative is to replace the absolute value by a smooth absolute value. The smoothed TV norm reads:

$$
\Pi(x)=\sum_{i} \sqrt{\|\nabla x(i)\|^{2}+\varepsilon^{2}}
$$

When $\varepsilon$ gets close to zero, the smoothed energy becomes closer to the original total variation, but the optimization becomes more difficult. When $\mathcal{E}$ becomes large, the smoothed energy becomes closer to the Sobolev energy, thus blurring the edges. Unfortunately, this prior is nonquadratic, and cannot be expressed over the Fourier domain. So an iterative scheme such as a gradient descent is used to approximate the solution.

For deblurring, initially, the deblurred image denoted by $x$ is initialized with the value of blurred image. By using the gradient descent optimization method, the value of $x$ is updated in each iteration. Gradient descent is implemented in TV with the following equation.

$$
x_{(k+1)}=x_{(k)}-\gamma\left(h *\left(h * x_{(k)}-y\right)+\lambda \operatorname{Grad} \Pi\left(x_{(k)}\right)\right)
$$

where $x_{(k+1)}$ and $x_{(k)}$ denotes the value of $x$ in the current and previous iterations respectively. This process continues until the stopping criteria are met. Here, the number of iterations is fixed which is considered as the stopping criteria. The deblurred image obtained with TV prior of blurred lena image is shown in Figure 5(e).

\subsection{Sparsity Prior}

Sparsity prior[4] considers a synthesis-based regularization, that compute a sparse set of coefficients $\left(a_{m}^{*}\right)_{m}$ in a frame $\Psi=\left(\psi_{m}\right)_{m}$ i.e., wavelet transform [19] is applied over the blurred image. After applying the wavelet transform, the energy will be present in lower number of coefficients (low-low band coefficients are taken). The L1-norm of the sparse set of coefficients is taken as the prior. The deblurred image $x$ can be obtained from

$$
x^{*}=\arg \min _{x} \frac{1}{2}\left\|y-h^{*} x\right\|^{2}+\lambda\left\|\left\langle x, \psi_{m}\right\rangle\right\|_{1}
$$

where $\left\|\left\langle x, \psi_{m}\right\rangle\right\|_{1}=\sum_{m}\left|\left\langle x, \psi_{m}\right\rangle\right|, y-$ the blurred image and $h$-blurring filter PSF. To solve this non-smooth optimization problem, iterative soft thresholding is used. It computes a series of images $x^{(l)}$ defined as 
The International Journal of Multimedia \& Its Applications (IJMA) Vol.4, No.1, February 2012

$$
x^{(l+1)}=S_{\tau \lambda}^{\psi}\left(x^{(l)}-\tau h^{*}\left(h * x^{(l)}-y\right)\right)
$$

where $\lambda$ is a constant which should be adapted to noise. For $x^{(l)}$ to converge to a solution of the problem, the gradient step size should be chosen as

$$
\tau<\frac{2}{\|h * h\|}
$$

Total number of iterations is set to 1000. Soft thresholding is performed for denoising. The deblurred image of blurred lena image using this sparsity prior is shown in Figure 5(f).

\subsection{Sparsity and Redundant Prior}

This prior considers that the contents can vary significantly across different images or different patches in a single image. The adaptive sparse domain selection (ASDS) method [5] uses this prior. This method learns various sets of bases from a pre-collected dataset of example image patches, and then for a given patch to be processed, one set of bases are adaptively selected to characterize the local sparse domain. Consider the Eq (20).

$$
x=\Phi \alpha
$$

where $\Phi=\left[\phi_{1}, \phi_{2}, \ldots, \phi_{m}\right]$ is a given dictionary of atoms (i.e., code set), $\alpha-$ the set of coefficients where most of coefficients are close to zero and $\boldsymbol{x}$, the deblurred image. With the sparsity prior, the representation of $\boldsymbol{x}$ over $\Phi$ can be estimated from its observation $\boldsymbol{y}$ by solving the following L0-minimization problem:

$$
\hat{\alpha}=\underset{\alpha}{\arg \min }\left\{\|y-H \Phi \alpha\|_{2}^{2}+\lambda\|\alpha\|_{0}\right\}
$$

where the L0-norm counts the number of nonzero coefficients in vector $\alpha$. Once $\hat{\alpha}$ is obtained, $\boldsymbol{x}$ can then be estimated as $\hat{x}=\Phi \hat{\alpha}$. The L0-minimization is an NP-hard combinatorial search problem, and is usually solved by greedy algorithms. Set of compact sub-dictionaries from high quality example image patches are learned using the principal component analysis (PCA) technique. For an image patch to be coded, the best sub-dictionary that is most relevant to the given patch is selected.

Suppose that $\left\{\phi_{k}\right\}, k=1,2, \ldots, K$, is a set of $K$ orthonormal sub-dictionaries. Let $\boldsymbol{x}$ be an image vector, $\boldsymbol{x}_{i}=\boldsymbol{R}_{i} \boldsymbol{x}$, and $i=1,2, \ldots, N$, be the $i$ th patch (size: $7 \times 7$ ) vector of $\boldsymbol{x}$, where $\boldsymbol{R}_{i}$ is a matrix extracting patch $\boldsymbol{x}_{i}$ from $\boldsymbol{x}$. For patch $\boldsymbol{x}_{i}$, suppose that a sub-dictionary $\phi_{k_{i}}$ is selected for it. Then, $\boldsymbol{x}_{i}$ can be approximated as $\hat{x}_{i}=\phi_{k_{i}} \alpha_{i},\left\|\alpha_{i}\right\|_{0} \leq T$ via sparse coding. The whole image $\boldsymbol{x}$ can be reconstructed by averaging all the reconstructed patches $\hat{x}_{i}$, which can be mathematically written as:

$$
\hat{x}=\left(\sum_{i=1}^{N} R_{i}^{T} R_{i}\right)^{-1} \sum_{i=1}^{N}\left(R_{i}^{T} \phi_{k_{i}} \alpha_{i}\right)
$$

The deblurred image of blurred lena image using the ASDS is shown in Figure 5(g).

\section{RESUlTS AND DISCUSSION}

Experiments were conducted on gray scale images [20], color images [20] and satellite images. The performance of the restoration process is quantified using Peak Signal-to-Noise Ratio (PSNR), Signal-to-noise ratio (SNR), Structural similarity index measure (SSIM) and Image 
The International Journal of Multimedia \& Its Applications (IJMA) Vol.4, No.1, February 2012

quality index (IQI). The PSNR, SNR, SSIM and IQI values obtained for 6 gray scale images blurred with Uniform and Gaussian blur for various parameter values are shown in tables 3 - 10 .

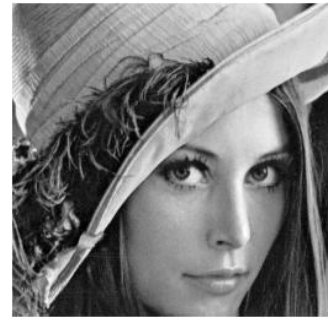

(a)

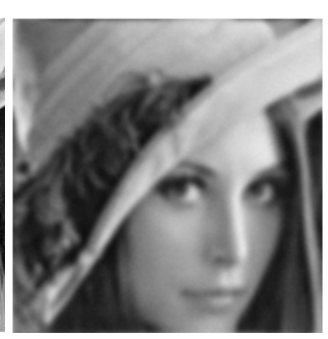

(b)

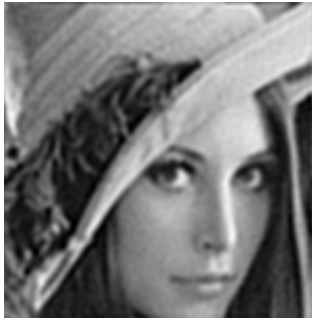

(c)

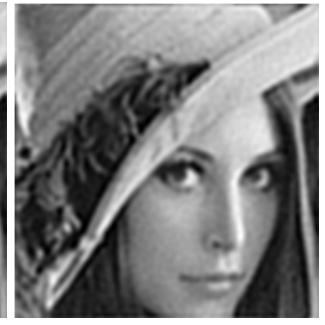

(d)

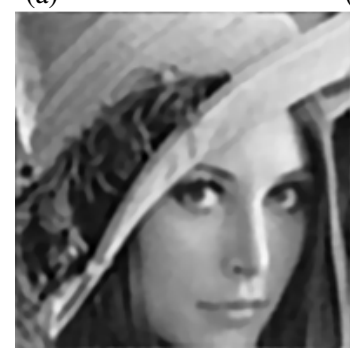

(e)

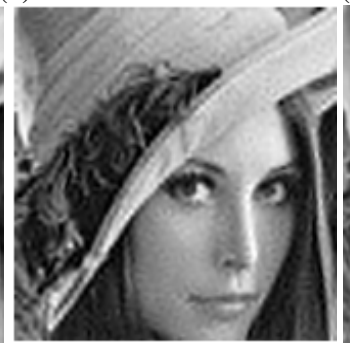

(f)

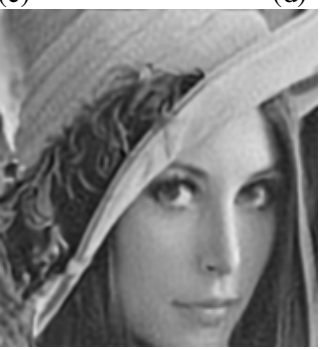

(g)

Figure 5. Restoration performed on lena image blurred by Gaussian blur of standard deviation 2.5 (a) Original Lena image (b) Gaussian blurred image with variance 2.5 (c) L2 (d) Sobolev (e) TV (f) Sparsity (g) ASDS
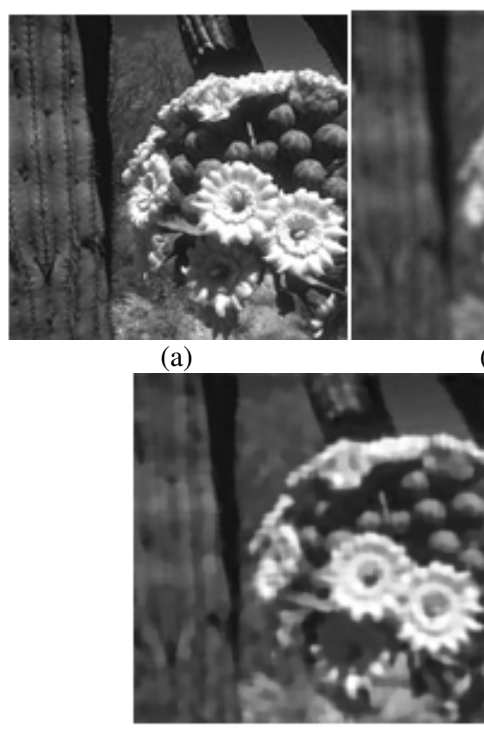

(e)

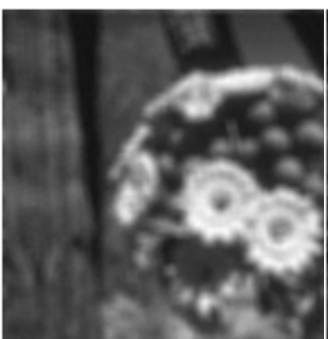

(b)
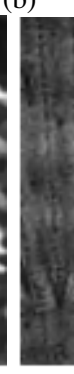

(f)

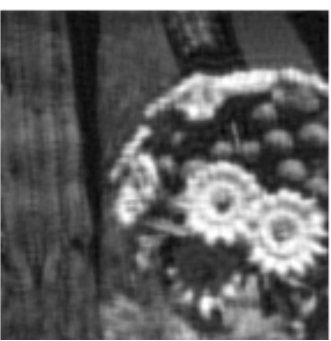

(c)

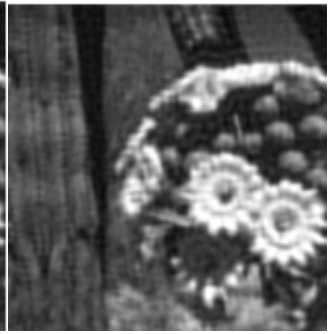

(d)

Figure 6. Restoration performed on cactus image blurred by Uniform blur of size 9x9 (a) Original image (b) Blurred image (c) L2 restored (d) Sobolev restored (e) TV restored (f) Sparsity (g) ASDS 
The International Journal of Multimedia \& Its Applications (IJMA) Vol.4, No.1, February 2012

Table 3. Table showing PSNR values in $\mathrm{dB}$ of the blurred and restored images blurred with Uniform kernel of size $9 \mathrm{x} 9$

\begin{tabular}{|c|c|c|c|c|c|c|}
\hline Name & Blurred & L2 & Sobolev & TV & Sparsity & ASDS \\
\hline boat & 20.37 & 23.91 & 24.45 & 24.60 & 26.02 & $\mathbf{2 9 . 9 5}$ \\
\hline cameraman & 19.34 & 22.72 & 23.04 & 23.45 & 25.48 & $\mathbf{2 9 . 2 6}$ \\
\hline lena & 21.32 & 24.12 & 24.54 & 24.67 & 25.79 & $\mathbf{2 9 . 7 7}$ \\
\hline cactus & 20.33 & 23.51 & 23.89 & 23.69 & 25.08 & $\mathbf{2 6 . 0 6}$ \\
\hline building & 18.56 & 21.27 & 21.49 & 21.36 & 23.67 & $\mathbf{2 5 . 3 2}$ \\
\hline animal & 21.76 & 24.63 & 24.96 & 24.72 & 25.84 & $\mathbf{2 7 . 0 9}$ \\
\hline
\end{tabular}

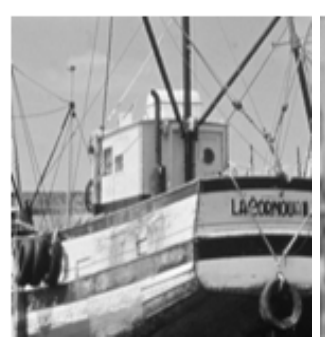

(a)

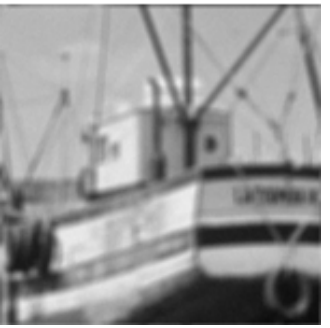

(b)

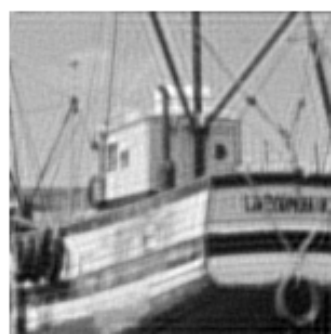

(c)

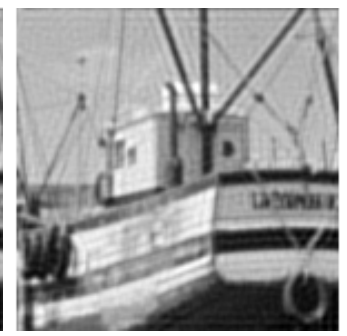

(d)

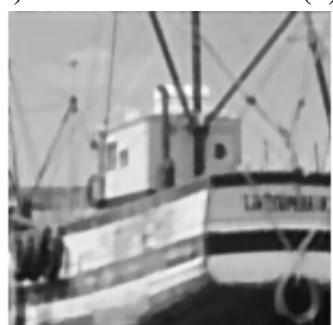

(e)

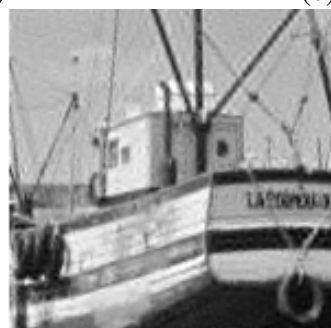

(f)

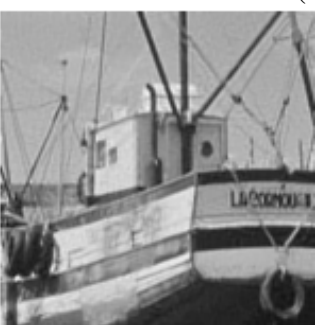

(g)

Figure 7. Restoration performed on boat image blurred by Uniform blur of size 7x7 (a) Original boat image (b) Blurred image (c) L2 restored (d) Sobolev restored (e) TV restored (f) Sparsity (g) ASDS

Table 4. Table showing SSIM values of restored images blurred with Uniform kernel size 9x9

\begin{tabular}{|c|c|c|c|c|c|}
\hline Name & L2 & Sobolev & TV & Sparsity & ASDS \\
\hline boat & 0.575 & 0.578 & 0.578 & 0.582 & $\mathbf{0 . 8 5 5}$ \\
\hline cameraman & 0.604 & 0.606 & 0.608 & 0.612 & $\mathbf{0 . 9 2 2}$ \\
\hline lena & 0.588 & 0.591 & 0.591 & 0.593 & $\mathbf{0 . 8 2 2}$ \\
\hline cactus & 0.572 & 0.574 & 0.574 & 0.577 & $\mathbf{0 . 7 8 2}$ \\
\hline building & 0.574 & 0.577 & 0.577 & 0.583 & $\mathbf{0 . 8 1 9}$ \\
\hline animal & 0.609 & 0.612 & 0.612 & 0.614 & $\mathbf{0 . 7 7 0}$ \\
\hline
\end{tabular}


The International Journal of Multimedia \& Its Applications (IJMA) Vol.4, No.1, February 2012

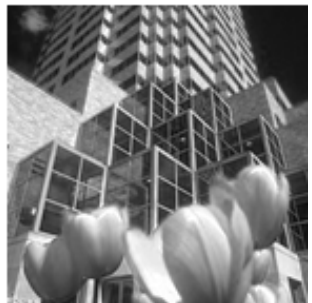

(a)

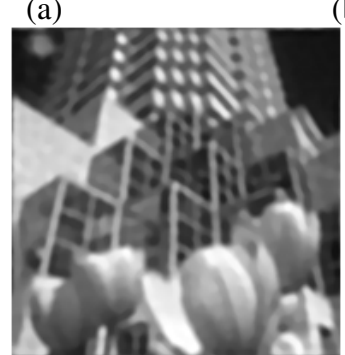

(e)

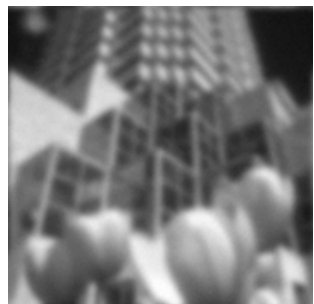

(b)

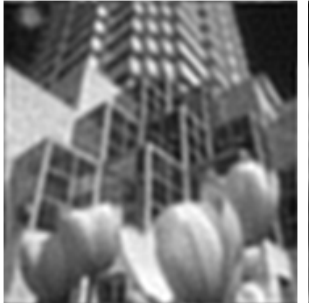

(c)

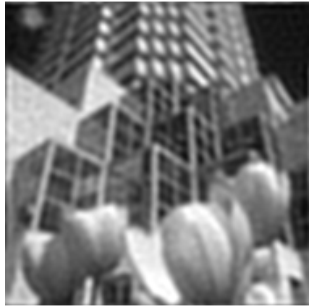

(d)

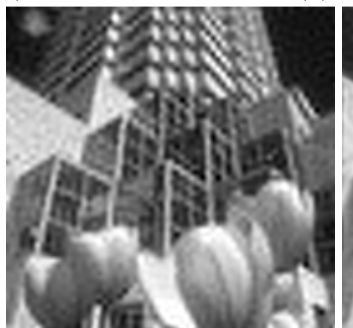

(f)

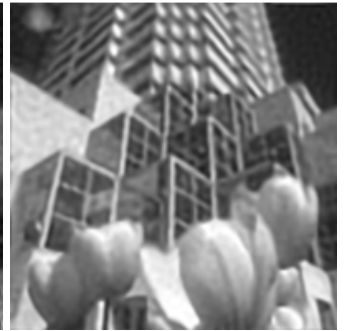

(g)

Figure 8. Restoration performed on building image blurred by Gaussian blur of standard deviation 2.9 (a) Original image (b) Blurred image (c) L2 restored (d) Sobolev restored (e) TV restored (f) Sparsity (g) ASDS

Table 5. Table showing SNR values of restored images blurred with Uniform kernel size 9x9

\begin{tabular}{|c|c|c|c|c|c|c|}
\hline Name & Blurred & L2 & Sobolev & TV & Sparsity & ASDS \\
\hline boat & 16.32 & 19.77 & 20.14 & 20.55 & 21.11 & $\mathbf{2 5 . 3 3}$ \\
\hline cameraman & 12.19 & 15.46 & 15.59 & 16.30 & 17.06 & $\mathbf{2 2 . 1 4}$ \\
\hline lena & 15.83 & 18.63 & 19.03 & 19.19 & 19.47 & $\mathbf{2 4 . 2 5}$ \\
\hline cactus & 12.84 & 15.45 & 15.71 & 15.74 & 16.11 & $\mathbf{1 8 . 8 1}$ \\
\hline building & 12.83 & 15.29 & 15.39 & 15.57 & 16.14 & $\mathbf{2 0 . 1 0}$ \\
\hline animal & 16.31 & 18.85 & 19.13 & 19.26 & 19.52 & $\mathbf{2 2 . 1 2}$ \\
\hline
\end{tabular}

Table 6. Table showing IQI values of restored images blurred with Uniform kernel size 9x9

\begin{tabular}{|c|c|c|c|c|c|}
\hline Name & L2 & Sobolev & TV & Sparsity & ASDS \\
\hline boat & 0.537 & 0.549 & 0.545 & 0.574 & $\mathbf{0 . 7 0 4}$ \\
\hline cameraman & 0.402 & 0.422 & 0.474 & 0.438 & $\mathbf{0 . 6 5 6}$ \\
\hline lena & 0.599 & 0.629 & 0.598 & 0.629 & $\mathbf{0 . 7 4 1}$ \\
\hline cactus & 0.584 & 0.601 & 0.569 & 0.611 & $\mathbf{0 . 7 3 7}$ \\
\hline building & 0.583 & 0.601 & 0.596 & 0.628 & $\mathbf{0 . 7 7 9}$ \\
\hline animal & 0.577 & 0.594 & 0.567 & 0.601 & $\mathbf{0 . 7 0 3}$ \\
\hline
\end{tabular}

Table 7. Table showing PSNR values in $\mathrm{dB}$ of the blurred and restored images blurred with Gaussian blur of standard deviation 2.5

\begin{tabular}{|c|c|c|c|c|c|c|c|}
\hline Name & Blurred & $\begin{array}{c}\text { Estimated } \\
\text { Variance }\end{array}$ & L2 & Sobolev & TV & Sparsity & ASDS \\
\hline boat & 21.43 & 2.36 & 23.53 & 24.13 & 24.10 & 25.78 & $\mathbf{2 6 . 8 8}$ \\
\hline cameraman & 20.39 & 2.34 & 22.42 & 22.92 & 22.85 & 25.25 & $\mathbf{2 5 . 5 8}$ \\
\hline lena & 22.24 & 2.3 & 24.35 & 24.69 & 24.85 & 25.84 & $\mathbf{2 8 . 4 8}$ \\
\hline cactus & 21.06 & 2.4 & 23.39 & 23.92 & 23.70 & 25.29 & $\mathbf{2 5 . 6 6}$ \\
\hline building & 19.33 & 2.5 & 20.90 & 21.34 & 21.11 & 23.07 & $\mathbf{2 3 . 4 5}$ \\
\hline animal & 22.54 & 2.4 & 24.32 & 24.85 & 24.68 & 26.06 & $\mathbf{2 6 . 4 5}$ \\
\hline
\end{tabular}


The International Journal of Multimedia \& Its Applications (IJMA) Vol.4, No.1, February 2012

Table 8. Table showing SSIM values of restored images blurred with Gaussian blur of standard deviation 2.5

\begin{tabular}{|c|c|c|c|c|c|}
\hline Name & L2 & Sobolev & TV & Sparsity & ASDS \\
\hline boat & 0.574 & 0.577 & 0.577 & 0.579 & $\mathbf{0 . 7 7 9}$ \\
\hline cameraman & 0.603 & 0.606 & 0.606 & 0.608 & $\mathbf{0 . 8 5 5}$ \\
\hline lena & 0.588 & 0.591 & 0.591 & 0.593 & $\mathbf{0 . 7 9 7}$ \\
\hline cactus & 0.572 & 0.574 & 0.574 & 0.576 & $\mathbf{0 . 7 1 7}$ \\
\hline building & 0.574 & 0.577 & 0.577 & 0.581 & $\mathbf{0 . 7 5 1}$ \\
\hline animal & 0.609 & 0.612 & 0.612 & 0.613 & $\mathbf{0 . 7 2 7}$ \\
\hline
\end{tabular}

Table 9. Table showing SNR values of restored images blurred with Gaussian blur of standard deviation 2.5

\begin{tabular}{|c|c|c|c|c|c|c|}
\hline Name & Blurred & L2 & Sobolev & TV & Sparsity & ASDS \\
\hline boat & 17.38 & 19.48 & 19.86 & 20.05 & 20.68 & $\mathbf{2 2 . 3 6}$ \\
\hline cameraman & 13.23 & 15.27 & 15.53 & 15.69 & 16.39 & $\mathbf{1 7 . 2 5}$ \\
\hline lena & 16.76 & 18.86 & 19.21 & 19.36 & 19.99 & $\mathbf{2 2 . 8 2}$ \\
\hline cactus & 13.67 & 15.57 & 15.81 & 15.90 & 16.15 & $\mathbf{1 7 . 4 1}$ \\
\hline building & 13.59 & 15.12 & 15.32 & 15.33 & 15.92 & $\mathbf{1 7 . 9 8}$ \\
\hline animal & 17.12 & 18.80 & 19.08 & 19.15 & 19.72 & $\mathbf{2 1 . 0 8}$ \\
\hline
\end{tabular}

Table 10. Table showing IQI values of restored images blurred with Gaussian blur of standard deviation 2.5

\begin{tabular}{|c|c|c|c|c|c|}
\hline Name & L2 & Sobolev & TV & Sparsity & ASDS \\
\hline boat & 0.553 & 0.563 & 0.547 & 0.570 & $\mathbf{0 . 6 2 1}$ \\
\hline cameraman & 0.480 & 0.484 & 0.541 & 0.466 & $\mathbf{0 . 5 8 6}$ \\
\hline lena & 0.657 & 0.668 & 0.652 & 0.669 & $\mathbf{0 . 7 2 0}$ \\
\hline cactus & 0.604 & 0.616 & 0.608 & 0.619 & $\mathbf{0 . 6 7 7}$ \\
\hline building & 0.616 & 0.628 & 0.628 & 0.647 & $\mathbf{0 . 7 1 9}$ \\
\hline animal & 0.601 & 0.611 & 0.598 & 0.616 & $\mathbf{0 . 6 6 5}$ \\
\hline
\end{tabular}

From these tables and resultant deblurred images (results obtained on lena, cactus, boat and building images are shown in figures $5-8$ ), it can infer that the sparse and redundant prior (ASDS method) gives better result. So for deblurring color images ASDS method is used and the deblurred images obtained are shown in figures 9 - 10, performance measure values obtained are shown in tables 11. For color images, all the test methods were applied to the luminance component only because human visual system is more sensitive to luminance changes, and the bicubic interpolator was applied to the chromatic components. Uniform blur kernel of size 9x9 is applied over the hat image to generate the blurred image. The blur type and its parameters are identified as uniform blur and 9 respectively. With this estimation, the blurring filter PSF for deblurring is constructed. The deblurred image with this estimated PSF is shown in Figure 9. Gaussian blur with standard deviation 2.5 is applied over the flower image to generate the blurred image. The blur type and parameters are identified as Gaussian blur and 2.4 respectively. With this estimation, the blurring filter PSF for deblurring is constructed, the deblurred image with this estimated PSF is shown in Figure 10. 
The International Journal of Multimedia \& Its Applications (IJMA) Vol.4, No.1, February 2012

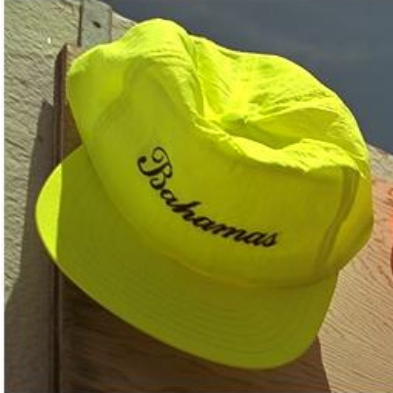

(a)

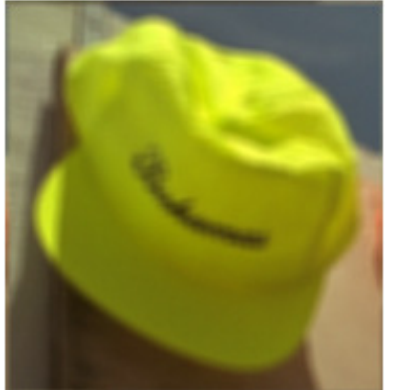

(b)

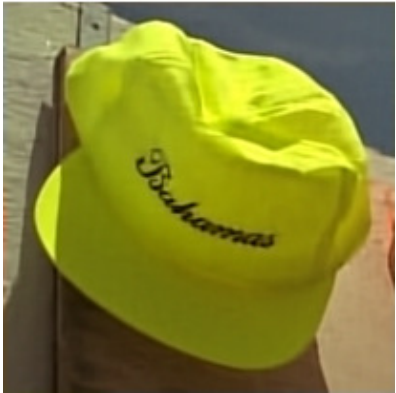

(c)

Figure 9. Restoration performed on hat image blurred by Uniform blur of size 9x9 (a) original hat image (b) Blurred image (c) Restored image

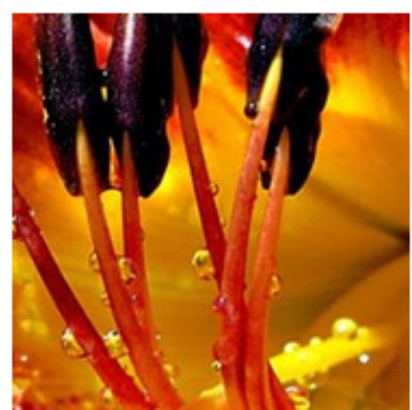

(a)

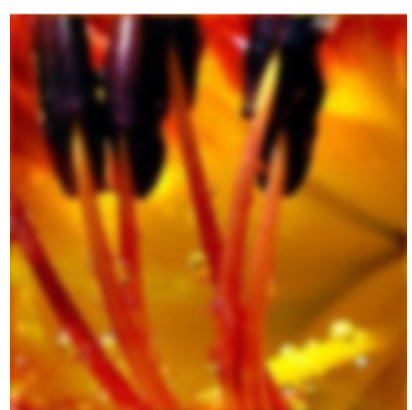

(b)

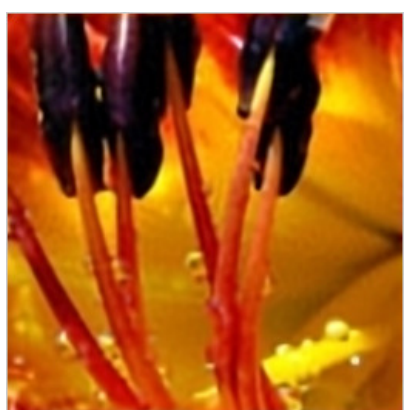

(c)

Figure 10. Restoration performed on flower image blurred by Gaussian blur of standard deviation 2.5 (a) original flower image (b) Blurred image (c) Restored image

Table 11. Table showing PSNR, SNR and SSIM values obtained for color images

\begin{tabular}{|c|c|c|c|c|c|c|c|}
\hline \multirow[b]{2}{*}{ Image } & \multirow[b]{2}{*}{$\begin{array}{l}\text { Blur type with } \\
\text { its parameter }\end{array}$} & \multicolumn{2}{|c|}{ Blurred image } & \multicolumn{4}{|c|}{ Restored image } \\
\hline & & PSNR & SNR & $\begin{array}{c}\text { Restoratio } \\
\text { n } \\
\text { Parameter }\end{array}$ & PSNR & SNR & SSIM \\
\hline Hat & Gaussian 2.8 & 25.74 & 18.11 & $\begin{array}{c}\text { Gaussian } \\
2.725\end{array}$ & 31.59 & 22.43 & 0.867 \\
\hline Parrot & Gaussian 2.5 & 24.33 & 17.32 & $\begin{array}{c}\text { Gaussian } \\
2.4\end{array}$ & 28.36 & 20.95 & 0.874 \\
\hline Flower & Uniform 9 & 24.86 & 17.13 & Uniform 9 & 29.67 & 20.66 & 0.867 \\
\hline Tiger & Uniform 7 & 22.72 & 13.38 & Uniform 7 & 29.77 & 19.85 & 0.868 \\
\hline
\end{tabular}

Naturally blurred satellite image due to atmospheric turbulence is deblurred where the blur type is estimated as Gaussian and its parameter as 1.3. The deblurred satellite image using various priors is shown in Figure 11. 
The International Journal of Multimedia \& Its Applications (IJMA) Vol.4, No.1, February 2012

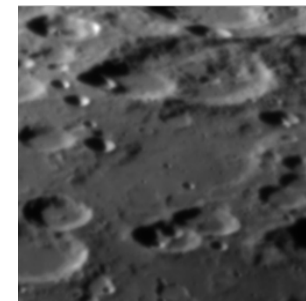

(a)

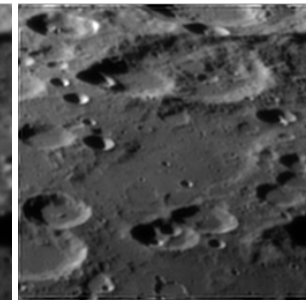

(b)

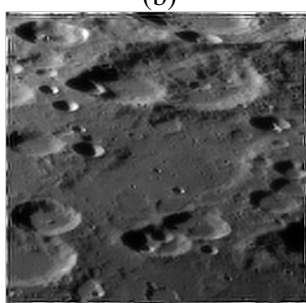

(e)

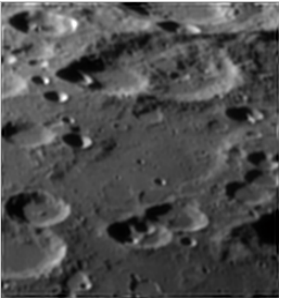

(c)

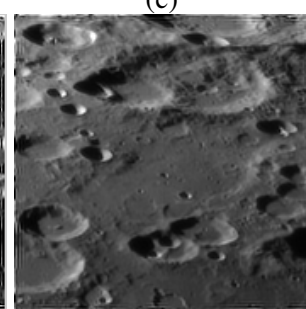

(f)

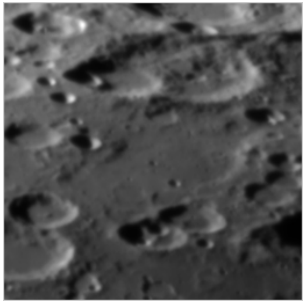

(d)

Figure 11. Restoration performed on blurred raw_moon1 image (a) original blurred raw_moon1 image (b) L2 restored (c) Sobolev restored (d) TV restored (e) Sparsity (f) ASDS

\section{Conclusions}

In this paper, a template based method for estimating the blur type and its parameter is proposed which can identify Gaussian and Uniform blur. The blurring filter PSF constructed from this estimation is used for deblurring the images. Image restoration is performed in the Bayesian framework where L2, Sobolev, Total variation, sparse, sparse and redundant priors are used. The performance measures used are Peak Signal-to-Noise Ratio (PSNR), Signal-to-noise ratio (SNR), Structural similarity index measure (SSIM) and Image quality index (IQI). Experiments were conducted with various blur parameters set for both Uniform blur and Gaussian blur on gray scale images as well as on color images. From the deblurred images obtained and performance measured used, it is inferred that the sparse and redundant prior gives better result. The system is also tested with naturally blurred moon images, visual comparison is done in this case, the sparse and redundant prior gave better result. Now the system can recognize and deblur Gaussian and Uniform blurred images only. Upgrading the system so that it can deblur images blurred by other blur models is the future scope.

\section{ACKNOWLEDGEMENTS}

I, on this occasion, remember the valuable suggestions and prayers offered by my family members and friends which were inevitable for the successful completion of this work.

\section{REFERENCES}

[1] D. Kundur and D. Hatzinakos, (1996) "Blind image deconvolution," IEEE Sig. Process. Mag., pp. 43-64.

[2] K. M. Hanson, (1993) "Introduction to Bayesian image analysis", SPIE Proceedings in ImageProcessing, pp 716-731.

[3] Jalal M. Radili and Gabriel Peyre, (2011) "Total Variation Projection with First Order Schemes", IEEE Transactions on Image Processing, Vol. 20, pp. 657-669 .

[4] D. L. Donoho, M.E Raimondo,(2004) " A fast wavelet algorithm for image deblurring", SIAM.

[5] Weisheng Dong, Lei Zhang et. al, (2011) "Image Deblurring and Super-resolution by Adaptive Sparse Domain Selection and Adaptive Regularization", IEEE Transactions on Image Processing. 
The International Journal of Multimedia \& Its Applications (IJMA) Vol.4, No.1, February 2012

[6] A. S. Carasso, (2001) "Direct blind deconvolution," SIAM J. Appl. Math.

[7] A. S. Carasso, (2002) "The APEX method in image sharpening and the use of low exponent Évy stable laws,” SIAM J. Appl. Math., vol. 63, no. 2, pp. 593-618.

[8] Y.-L. You and M. Kaveh,(1999) "Blind image restoration by anisotropic regularization," IEEE Trans. Image Process., vol. 8, no. 3, pp. 396-407.

[9] R. Molina, J. Mateos, and A. K. Katsaggelos, (2006) "Blind deconvolution using a variational approach to parameter, image, and blur estimation," IEEE Trans. Image Process., vol. 15, no. 12, pp. 3715-3727.

[10] Y.-L. You and M. Kaveh, (1996) "A regularization approach to joint blur identification and image restoration,” IEEE Trans. Image Process., vol. 5, no. 3, pp. 416-428.

[11] T. F. Chan and C.-K. Wong, (1998) "Total variation blind deconvolution," IEEE Trans. Image Process., vol. 7, no. 3 .

[12] L. He, A. Marquina, and S. J. Osher, (2005) "Blind deconvolution using TV regularization and bregman iteration,” Int. J. Imag. Syst. Technol., vol. 15, pp. 74-83.

[13] A. Kubota and K. Aizawa, (2005) "Reconstructing arbitrarily focused images from two differently focused images using linear filters," IEEE Trans. Image Process., vol. 14, no. 11, pp. 1848-1859.

[14] V. Katkovnik, K. Egiazariana, and J. Astola, (2005) "A spatially adaptive nonparametric regression image deblurring," IEEE Trans. Image Process., vol. 14, no. 10, pp. 1469-1478.

[15] Mariana S. C. Almeida and Luís B. Almeida, (2010) "Blind and Semi-Blind Deblurring of Natural Images," IEEE Trans. Image Process. Vol. 19, no. 1.

[16] R.Lokhande, K. V. Arya and P. Gupta, (2006) "Identification of Parameters and Restoration of Motion Blurred Images," ACM SAC' 06.

[17] Rafael C. Gonzalez and Richard E. Woods, (2009) "Digital Image Processing”, in Prentice Hall.

[18] Gose Earl, Johnson Baugh Richard and Jost Steve, (1997) "Pattern Recognition and Image Analysis", NewDelhi, Prentice Hall of India.

[19] http://paos.colorado.edu/research/wavelets/

[20] http://www.eecs.berkeley.edu/Research/Projects/CS/vision/grouping/segbench 
The International Journal of Multimedia \& Its Applications (IJMA) Vol.4, No.1, February 2012

\section{Authors}

Sitara K was born in Kerala, India on December 5, 1987. She graduated in Computer Science \& Engineering from College of Engineering, Thiruvananthapuram, India in 2009. She did her masters at Universityof Kerala with specialization in Digital Image Computing. Her area of interest are image processing, operating systems and information retrieval.

Remya $\mathrm{S}$ was born in Kerala, India. Currently working as Lecturer in Department of Computer Science, University of Kerala. Handling classes for MTech Computer Science (with Specialization in Digital Image Processing).Subjects handling are Knowledge Based System, Geographic Information System, Embedded System, Networks, Object Oriented Software Engineering. Also guiding MTech students in their Dissertation works.
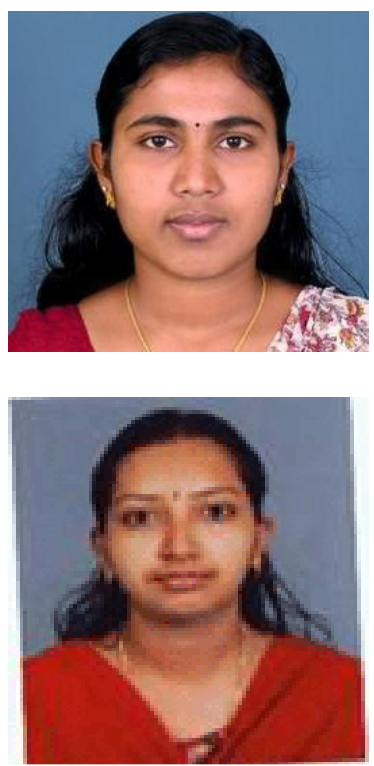